\title{
Highly diluted natural complex (M2) effects on leishmanias
}

\author{
Katia Fialho do Nascimento, Jenifer Pendiuk Gonçalves, Maurício Baum, \\ Izanara Pritsch, Magda Clara Vieira da Costa Ribeiro, Edilene Alcântara de \\ Castro, Carolina Camargo de Oliveira, Dorly de Freitas Buchi
}

\author{
Federal University of Paraná, Curitiba, Paraná, Brazil
}

Introduction: Leishmaniosis is regarded as a serious public health issue either by its magnitude, morbidity or mortality. Depending on Leishmania species disease ranges from cutaneous, which is relatively confined and controlled, until a progressive and fatal visceral disease [4]. In order to complete their lifecycle Leishmania undergo transformations in the vector digestive tract to get to metacyclic infective form $[3,5]$. Those include secretion of the "promastigote secretory gel" (PSG) that protect parasites from digestive enzymes. PSG acts on survival and colonization of parasites inside the vector, on transmission and infection development in mammals, and also facilitates and increases transmission [1,2]. Drugs used for leishmaniosis treatment show high toxicity and several side effects, leading patients to quit the treatment and consequently generating resistant strains. The search for new therapeutic approaches is considered a strategic research priority by the World Health Organization. Highly diluted natural products show efficacy in modifying immunological response by stimulating the immune system through macrophages activation, then favoring the organism in many pathological conditions. Aim: To assess the direct action of M2 treatment on promastigotes of different Leishmania species. Methods: 3x106 were cultured in the presence of 20\% of M2 plus booster $1 \%$ doses every $24 \mathrm{~h}$ for up to $96 \mathrm{~h}$ at $25^{\circ} \mathrm{C}$ in humidified incubator and then submitted to assays for determining mitochondrial activity by MTT $(5 \mathrm{mg} / \mathrm{mL})$, cell proliferation trough cell counting on Neubauer chamber and rosettes formation (around PSG) by light microscopy. Results of 3 independent experiments were statistically analyzed using t-test. Results: M2 treatment changed mitochondrial metabolic activity in all tested Leishmania strains. L. amazonensis proliferation decreased after $96 \mathrm{~h}$ treatment and likewise the rosettes formation (total, closed and open ones). Discussion: Our data show that Leishmania cultures treated with M2 present decreased number of rosettes and these rosettes may be slowing the production of PSG, that is typically synthesized by Leishmania during metacyclic infective phase. Rosettes are also a place for fusion between 2 or more leishmanias, a process that involves nuclear and kinetoplast genetic material exchange. Conclusion: M2 acts on L. amazonensis promastigote forms by reducing the total number of rosettes (the same for open and closed rosettes) that are related to infective form of promastigotes, which produce PSG while on rosettes. This result suggests that M2 treatment is capable of decreasing Leishmania infectivity. Although our results are preliminary, these changes open new perspectives for the disease treatment and/or prevention using M2.

Keywords: promastigote secretory gel, infectivity, proliferation, rosettes.

\section{References:}

[1] Bates PA. Transmission of Leishmania metacyclic promastigotes by phlebotomine sand flies. International $\mathrm{J}$ for Parasitology, 37 1097-1106, 2007.

[2] Bates PA, Rogers ME. Chance ML. The role of promastigote secretory gel in the origin and transmission of infective stage of Leishmanina mexicana by the sandfly Lutzomya longipalpis. Parasitology, 2001. 
[3] Kamhawis S. Phlebotomine sand fly and Leishmania parasites: friends or foes? Trends in Parasitology. Vol.22 n 9, 2006.

[4] Murray HW, Berman JD. Davis CR. Saraiva NG. Advances in Leishmaniasis. Lancet 2005; 366: 1561-77.

[5] Ramalho-Ortigão M, Saraiva EM, Traub-Cseko M. Sand fly - Leishmania Interations: Long Relationship are not necessarily Easy. The open Parasitology Journal, 2010, 4, 195-204.

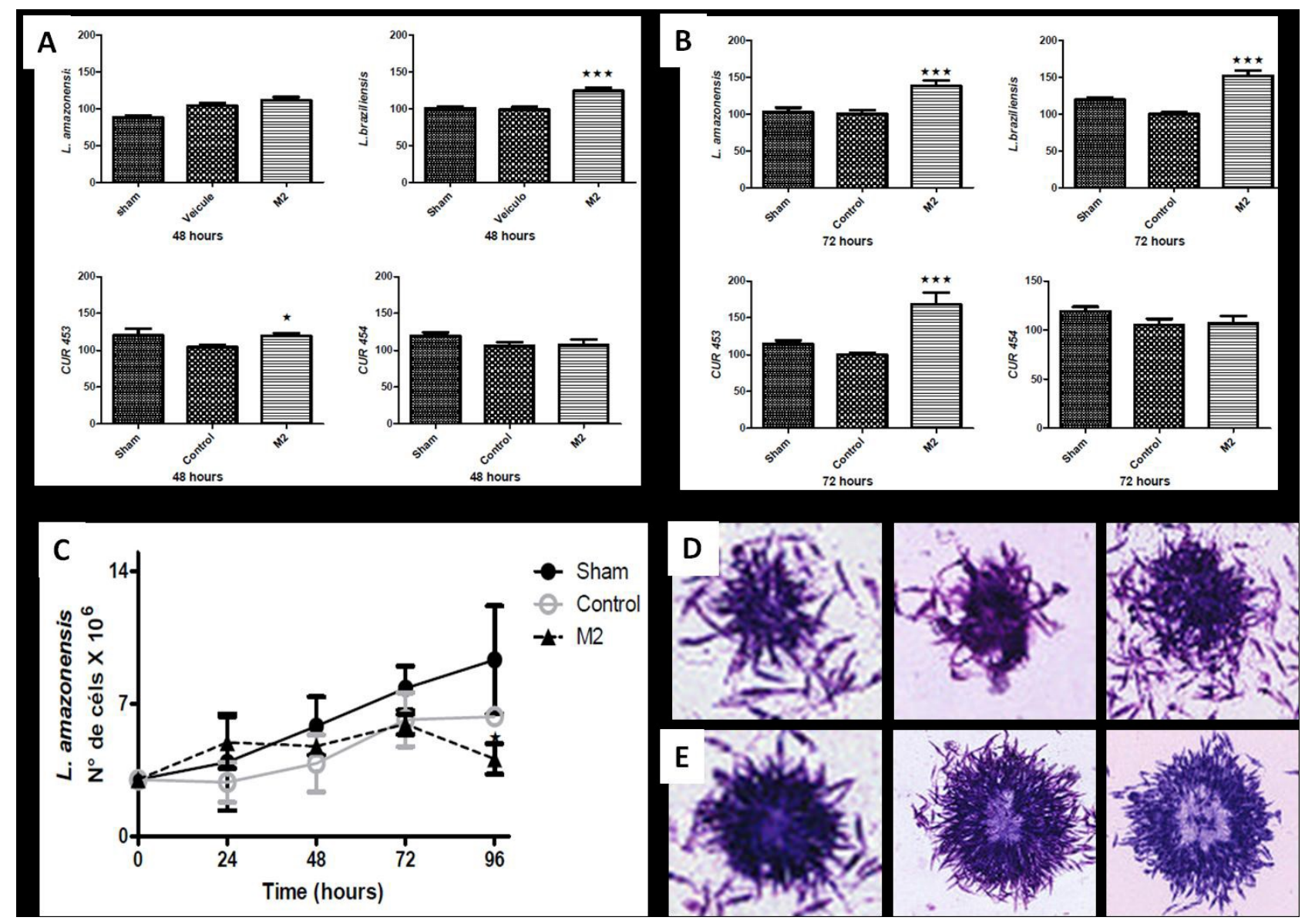

Figure 1. M2 treatment changed mitochondrial metabolic activity, cell proliferation and rosettes formation in Leishmania. Graphs A and B represent mitochondrial activity of the strains L. amazonensis, L. braziliensis, Cur 453 (L. amazonensis) and Cur 454 (L. braziliensis) treated with M2 for 48 and 72h. Graph C shows cell proliferation of L. amazonensis. The images illustrate what we considered as closed (D) and open rosettes (E), which were stained using May Grünwald and Giemsa.

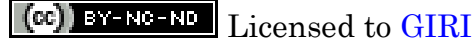

Support: This work was financially supported by CNPq, CAPES, Fundação Araucária. We had full access to all the data in this study and we take complete responsibility for the integrity of the data and the accuracy of the data analysis.

Conflict of interest: authors declare there is no conflict of interest

Received: March 30th 2014; Revised: May 10 ${ }^{\text {th }}$ 2014; Published: June 30 2014.

Correspondence author: Dorly de Freitas Buchi, buchi@ufpr.br dorlybuchi@gmail.com

How to cite this article: Nascimento KF, Gonçalves JP, Baum M, Pritsch I, Ribeiro MCVC, de Castro EA, de Oliveira CC, Buchi DF. Highly diluted natural complex (M2) effects on leishmanias. Int J High Dilution Res [online]. 2014 [cited YYYY Month dd]; 13(47):115-116. Proceedings of the XXVIII GIRI Symposium; 2014 Jun 20-22; Sighisoara (Romania). GIRI; 2014; Available from: http://www.feg.unesp.br/ ojs/index.php/ijhdr/article/view/729/706 